\title{
Factors related to RNA polymerase II transcription are localized in interchromatin granule clusters of Panorpa communis oocytes
}

\author{
Florina Batalova and Vladimir Parfenov
}

Institute of Cytology, Russian Academy of Sciences, St. Petersburg, Russia

\begin{abstract}
Diplotene oocyte nucleus of the scorpionfly Panorpa communis is transcriptionally silent and contains numerous nuclear bodies including interchromatin granule clusters (IGCs). The latter consist of the granules of 30-50 nm in diameter and contain IGC marker protein SC35 as well as RNA polymerase II. In this study, we also localized in P. communis oocyte IGCs the transcription coactivators CBP/p300, TATA-binding protein (TBP) which is a component of the basal transcription factor TFIID and the basal transcription factor TFIIH. We belive that IGCs in transcriptionally inert P. communis oocytes are storage sites for the components of RNA polymerase II holoenzyme and other factors of RNA pol II transcription.
\end{abstract}

Key words: insects, Panorpa communis, oocyte nucleus, interchromatin granule clusters, immunoelectron microscopy, RNA polymerase II, transcription factors.

\section{Introduction}

Interchromatin granules clusters (IGCs) also referred to as 'speckles' together with Cajal bodies (CB) and nucleoli are considered to be universal extrachromosomal nuclear domains for different cell types [1]. The nature of some characteristic inclusions observed in oocytes of different insects as the CBs are established now [2-5] whereas until recently little is known about insect oocyte IGC structure and functions. Insect oocyte IGCs have been described only in a beetle [6,7], the house cricket [8], a fleshfly [9] and some bugs [10]. In our previous study, we showed some features of IGC structure and molecular composition in oocyte nuclei of the scorpionfly, Panorpa communis [11]. Importantly, we found that along with splicing factor SC35, a marker protein of IGCs $[12,13]$, P. communis oocyte IGCs contain two (unphosphorylated and hyperphosphorylated) forms of RNA polymerase II (Pol II) [11]. In the present study, we extend these data attempting to localize in the IGCs of $P$. communis oocytes additionally to Pol II some other components of Pol II holoenzyme and

Correspondence: F. Batalova, Institute of Cytology RAS, Tikhoretsky Ave 4, 194064, St. Petersburg, Russian Federation; tel.: (+7812) 2976301, fax: (+7812) 2970341,

e-mail: florina@mail.cytspb.rssi.ru factors involved in activation of Pol II transcription. It is known that Pol II holoenzyme consists of core enzyme Pol II, general transcription factors, and the core Srb-mediator complex $[14,15]$. It should be noted that the participation of IGCs in nuclear distribution of the Pol II holoenzyme components remains to be more learned particularly in such highly specific cells as oocytes. Besides, the rare studies on this problem have been predominantly concerned the active state of the oocyte nucleus [16]. Therefore, it would be interesting to trace a behaviour of Pol II holoenzyme components in association with the IGCs in transcriptionally inert oocytes. It is the reason for choosing of $P$. communis diplotene oocytes as an experimental model, because they possess a fully inactivated nuclei at this stage of oogenesis when chromosome condensation and karyosphere formation occur $[11,17,18]$. Thus, using immunogold labeling/electron microscopy we have mapped inside the diplotene oocyte IGCs of $P$. communis the following component of Pol II holoenzyme: Pol II itself and basal transcription factors, TFIID and TFIIH. Additionally, we have defined the localization of transcriptional coactivators, $\mathrm{CBP} / \mathrm{p} 300$, in oocyte IGCs of this species.

\section{Material and methods}

The specimens of the scorpionfly, Panorpa communis L. were collected in June in the village of Toksovo (Leningrad region, Russia). 

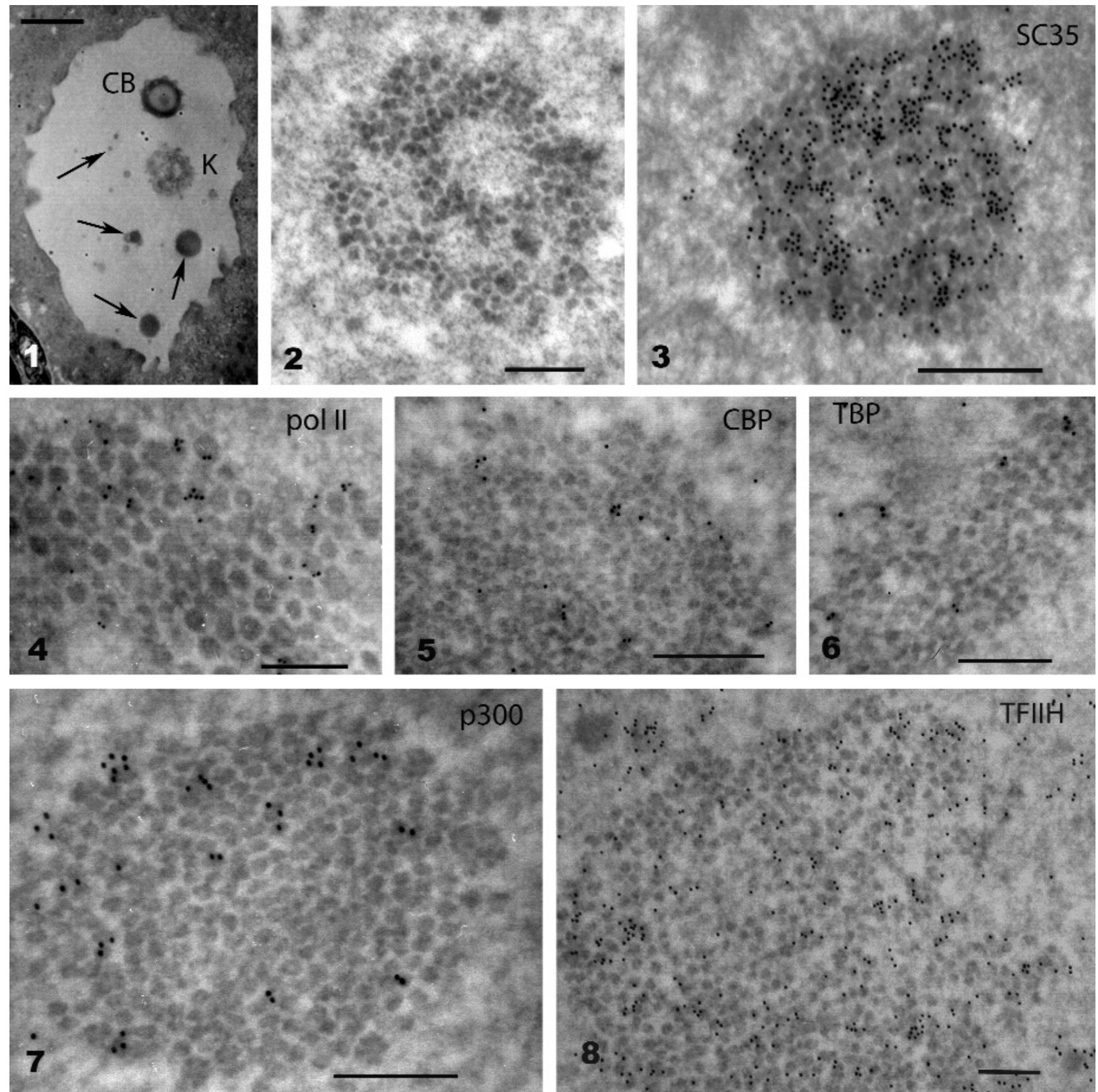

Fig. 1. A semithin section of Panorpa communis diplotene oocyte nucleus at the stage of late previtellogenesis. The largest nuclear body is the Cajal body (CB). Nuclear bodies (arrows) and a karyosphere $(\mathrm{K})$ are seen in the nucleoplasm. Bar $=5 \mu \mathrm{m}$. Fig. 2. Interchromatin granule cluster of $P$. communis oocytes as viewed by routine electron microscopy. Bar $=0.5 \mu \mathrm{m}$. Fig. 3-8. Interchromatin granule clusters of $P$. communis oocytes after immunogold labeling with antibodies against non-snRNP splicing factor SC35 (Fig. 3), hyperphosphorylated C-terminal domain (CTD) of the Pol II (Fig. 4), C-terminal domain of transcription coactivator CBP (Fig.5), full length TATAbinding protein (TBP) (Fig.6), N-terminal domain of transcription coactivator p300 (Fig. 7), and basal transcription factor TFIIH (Fig.8). Bar $=0.5 \mu \mathrm{m}$.

Ovarioles were prepared for immunogold labeling electron microscopy as described [11]. Primary antibodies included the following monoclones: $\alpha \mathrm{SC} 35$ against the non-snRNP splicing factor SC35 [12], V22 against the hyperphosphorylated C-terminal domain (CTD) of the Pol II, and the following rabbit polyclonal sera: N-17 against the component of the basal transcription factor TFIIH (Santa Cruz Biotechnology, Inc.), N-15 against the N-terminal domain of transcription coactivator p300 (Santa
Cruz Biotechnology, Inc.), SI-1 against the full length TATAbinding protein (TBP) (Santa Cruz Biotechnology, Inc.), N-20 against the $\mathrm{C}$-terminal domain of transcription coactivator CBP (Santa Cruz Biotechnology, Inc.).

Secondary antibodies were goat anti-mouse or goat anti-rabbit antibodies conjugated with colloidal gold particles of $10 \mathrm{~nm}$ (BBInternational). 


\section{Results and discussion}

The nuclear morphology of $P$. communis oocytes presented here corresponds to that described earlier [11]. The general appearance of diplotene oocyte nucleus at the stage of late previtellogenesis is shown in Fig. 1. The nucleus contains a complex of the karyosphere consisting of highly condensed chromosomes and numerous nuclear bodies (NBs); some of them are also distributed in nucleoplasm. In the previous study, the largest NB was identified as a (CB) [11]; other NBs composed of a granular material (Fig. 2) contain the marker component of IGCs - SC35 (Fig. 3) and, thus, may be reffered to as $P$. communis oocyte IGCs. The granules of $P$. communis oocyte IGCs range between $30-50 \mathrm{~nm}$, i. e. they are larger than those in the canonical IGCs of mammalian somatic cells where they are 20-25 nm in diameter [19]. Immunogold labeling with anti-SC35 antibody shows that all P. communis oocyte IGCs significantly accumulate IGC marker protein SC35 (Fig. 3). The IGCs were also labeled with antibodies against the Pol II (Fig. 4), transcriptional coactivators CBP (Fig.5) and p300 (Fig. 7), and both basal transcription factors TBP (Fig. 6) and TFIIH (Fig. 8). It should be mentioned that the experiments with all these antibodies revealed the same pattern of labeling: the labels were predominantly associated with the granules of IGC. The revealed association of Pol II holoenzyme component (Pol II and basal transcription factors) with IGCs granules of inactive oocytes is significant. In transcriptionally active cells, these data could be explained in the frame of the suggestion that each granule, or "Pol II transcriptosome", contains Pol II holoenzyme prior the transfer of a transcriptosome to active chromatin as it was postulated for the granules of Xenopus oocyte CBs [16]. At the same time, Xenopus oocyte IGCs (B-snurposomes) do not contain Pol II [20]. However, P. communis oocyte IGCs are unusual IGCs because they also contain the CB marker protein coilin [11] and, thus, may share some features of both IGCs and CBs. It should be also noted that $P$. communis diplotene oocytes used in this study are transcriptionally inert [17] and, in contrast to the active cells, the IGC granules of $P$. communis oocytes could be the storage sites for the Pol II holoenzyme components disengaged from transcription. The same function of IGCs in inactive insect oocytes was discussed earlier $[6,8]$. Additionally, our data on tight association of transcriptional coactivators $\mathrm{CBP} / \mathrm{p} 300$ with granules of IGCs in P. communis oocytes (Fig. 5, 7) suggest the $\mathrm{CBP} / \mathrm{p} 300$ which is not a part of the holoenzyme, does connect with the transcriptosomes of IGCs in inactive oocyte nuclei. In this case, the presence of the $\mathrm{CBP} / \mathrm{p} 300$ in IGCs is consistent with observations on the accumulation of $\mathrm{CBP} / \mathrm{p} 300$ in IGCs of some cells when Pol II transcription was inhibited by drugs [21].
Acknowledgements: The authors are indebted to the following people providing us with antibodies: J.G. Gall $(\alpha \mathrm{SC} 35)$ and U. Scheer (V22). We also thank Dr. D. Bogolyubov for collecting the specimens and critical reading of the manuscript and Yu. I. Gukina for technical assistance. This work was supported by Russian Foundation for Basic Research (grant No. 06-04-48904, 09-04-00723) and by the grant program "Molecular and Cellular Biology".

\section{References}

[ 1] Gall JG, Wu Z, Murphy C, Gao H. Structure in the amphibian germinal vesicle. Exp Cell Res. 2004;296:28-34.

[2] Gall JG, Tsvetkov A, Wu Z, Murphy C. Is the sphere organelle/coiled body a universal nuclear component? Dev Genet. 1995; 16:25-35.

[3] Jaglarz MK. Nuclear bodies in the oocyte nucleus of ground beetles are enriched in snRNPs. Tissue Cell. 2001;33:395-401

[4] Liu JL, Buszczak M, Gall JG. Nuclear bodies in the Drosophila germinal vesicle. Chromosome Res. 2006;14:465475.

[ 5] Biliński SM, Kloc M. Accessory nuclei revisited: the translocation of snRNPs from the germinal vesicle to the periphery of the future embryo. Chromosoma. 2002;111:62-68.

[ 6] Bogolyubov DS, Alexandrova O, Tsvetkov A, Parfenov VN. An immunoelectron study of karyosphere and nuclear bodies in oocytes of mealworm beetle, Tenebrio molitor (Coleoptera: Polyphaga). Chromosoma. 2000;109:415-425.

[ 7] Bogolyubov DS, Parfenov VN. Immunogold localization of RNA polymerase II and pre-mRNA splicing factors in Tenebrio molitor oocyte nuclei with special emphasis on karyosphere development. Tissue and Cell. 2001;33:549561.

[ 8] Stepanova IS., Bogolyubov DS., Skovorodkin IN., Parfenov VN. Cajal bodies and interchromatin granule clusters in cricket oocytes: composition, dynamics and interactions. Cell Biol. Int. 2007;31:203-214.

[ 9] Bogolyubov DS, Stepanova IS. Interchromatin granule clusters in vitellogenic oocytes of the fleshfly, Sarcophaga sp. Folia Histochem Cytobiol 2007;45:401-403.

[10] Bogolyubov DS, Batalova FM, Ogorzałek A. Localization of interchromatin granule cluster and Cajal body components in oocyte nuclear bodies of the hemipterans. Tissue and Cell. 2007;39:353-364.

[11] Batalova FM, Stepanova IS, Skovorodkin IN, Bogolyubov DS, Parfenov VN. Identification and dynamics of Cajal bodies in relation to karyosphere formation in scorpionfly oocytes. Chromosoma. 2005;113:428-439.

[12] Fu X-D, Maniatis T. Factor required for mammalian spliceosome assembly is localized to discrete regions in the nucleus. Nature. 1990;343:437-441.

[13] Spector DL, Fu X-D, Maniatis T. Associations between distinct pre-mRNA splicing components and the cell nucleus. EMBO J. 1991;10:3467-3481.

[14] Koleske AJ., Young RA. The RNA polymerase II holoenzyme and its implications for gene regulation. Trends Biochem. Sci.1995;20:113-116.

[15] Lee TI, Young RA. Transcription of eukaryotic protein-coding genes. Annu. Rev. Genet. 2000;34:77-137.

[16] Gall JG, Bellini M., Wu Z, Murphy C. Assembly of the nuclear transcription and processing machinery: Cajal bodies (coiled bodies) and transcriptosomes. Mol Biol Cell. 1999; 10:4385-4402.

[17] Bogolyubov DS. Localization of RNA transcription sites in insect oocytes using microinjections of 5-bromouridine 5'triphosphate. Folia Histochem Cytobiol. 2007;45:129-134. 
[18] Gruzova MN, Parfenov VN. Karyosphere in oogenesis and intranuclear morphogenesis. Int Rev Cytol. 1993;144:1-52

[19] Monneron A, Bernhard W. Fine structural organization of the interphase nucleus in some mammalian cells. $J$ Ultrastruct Res. 1969;27:266-288

[20] Doyle O, Corden JL, Murphy C, Gall JG. The distribution of RNA polymerase II largest subunit (RPB1) in the Xenopus germinal vesicle. J Struct Biol. 2002;140:154-166.
[21] von Mikecz A, Zhang S, Montminy M, Tan EM, Hemmerich P. CREB-binding protein (CBP)/p300 and RNA polymerase II colocalize in transcriptionally active domains in the nucleus. J Cell Biol. 2000;150:265-274.

Submitted: 7 July, 2008 Accepted after reviews: 12 October, 2008 